2017-10-28

\title{
Physical activity and mental health; it is more than just a prescription
}

Rebar, AL

http://hdl.handle.net/10026.1/10591

10.1016/j.mhpa.2017.10.004

Mental Health and Physical Activity

Elsevier

All content in PEARL is protected by copyright law. Author manuscripts are made available in accordance with publisher policies. Please cite only the published version using the details provided on the item record or document. In the absence of an open licence (e.g. Creative Commons), permissions for further reuse of content should be sought from the publisher or author. 
Running Head: MENTAL HEALTH AND BEHAVIOR CHANGE

Physical Activity and Mental Health; It is More than Just a Prescription

\author{
Amanda $\operatorname{Rebar}^{1} \&$ Adrian Taylor ${ }^{2}$
}

Editorial for Special Issue on behavior change processes

1 School of Human Health \& Social Sciences, Central Queensland University, Australia

2. Plymouth University Schools of Medicine \& Dentistry, UK 


\begin{abstract}
Most mental health and physical activity research describes unidirectional causes of physical activity on mental health, and as a result, a strong evidence base is being established for the effectiveness of physical activity as a treatment for mental health issues. Given that the efficacy of physical activity prescriptions are entirely reliant on individuals' behavioral engagement, the aim of this special issue is to draw attention to translational evidence relevant to mental health and physical activity. This issue encompasses findings from a wide array of study designs (e.g., reviews, qualitative investigations, correlations studies, trial descriptions, pilot trial findings) of populations from high, middle, and low-income countries with clinical and non-clinical mental health issues. The evidence illustrates that people with mental health issues have unique facilitators and barriers to physical activity that are not accounted for within behavior change theories or interventions for the general population. Within this issue, you will find evidence of how mental health issues impact physical activity behavior change processes as well as examples of how context and person factors may moderate physical activity intervention efficacy amongst these populations. Informed by this evidence, we are calling for future research to investigate acceptability, maintenance, scalability, and generalizability of physical activity interventions for people with mental health issues. This future research will need to account for the unique barriers and facilitators of the population, be theoretically sound, apply to unique contexts, and adapt to dynamic change processes (including engagement and maintenance).
\end{abstract}

Keywords: Trial efficacy; depression; anxiety; severe mental illness; substance abuse; exercise 
Physical Activity and Mental Health: It is More than Just a Prescription

Highlights

- This issue calls to attention the need for translational and efficacy evidence

- Mental health issues are associated with unique activity facilitators and barriers

- Mental health issues impact physical activity behaviour change processes

- Context, and person factors can impede or aide in behaviour change processes

- We need to investigate acceptability, maintenance, scalability and generalizability 
Mental health has reached unprecedented worldwide attention; for the first time, the promotion of mental health and the prevention and treatment of mental illnesses and substance abuse is included in the United Nations' Sustainable Development Goals (World Health Organization, 2017). Physical activity promotion may be a key strategy for enhancing mental health worldwide, given the strong and growing evidence that physical activity has mental health and wellbeing benefits for non-clinical (Rebar, Stanton, et al., 2015) and clinical (Bailey, Hetrick, Rosenbaum, Purcell, \& Parker, 2017; Rosenbaum, Tiedemann, Sherrington, Curtis, \& Ward, 2014) populations. Despite this evidence of efficacy, there remains a translational gap - we need to demonstrate the effectiveness of different interventions in engaging and treating people with mental health issues in the real world. Effectiveness is not only large-scale effectiveness trials though. An essential, but oftentimes neglected, part of the process of filling this translation gap is to establish how contextual and person factors affect behavioral engagement and intervention effectiveness.

The field of physical activity and mental health is generally lacking this translational evidence. There is evidence that people with mental health conditions are less active than the general population (e.g., Schuch et al., 2017) but very little is understood about how mental health issues may interfere with the psychological processes through which people initiate and maintain physical activity engagement (e.g., Farholm \& Sørensen, 2016; Roessler, Bramsen, Dervisevic, \& Bilberg, 2017). Additionally, little is known about how contextual or person factors impact these behavior change processes or the efficacy of interventions. Without this evidence, we risk wasting resources and time trying to fit mismatched interventions across unique populations and individuals. With this evidence, however, we can design evidence-based interventions to custom fit individuals' unique situations and their dynamic motivational processes, maximizing their potential mental health benefits. Our aim for this special issue for Mental Health and Physical Activity is to advance this agenda by 
providing researchers, service users, practitioners, and policy makers with a better understanding of behavior change processes for people with mental health issues and the contextual and person factors that may impact them. Within this editorial, we will introduce the articles within the special issue, present our reflections on how this new evidence should inform practice and intervention translation, and highlight existing gaps in our understanding as a strategic direction for future research.

\section{Mental Health Issues \& Physical Activity Behavior Change Processes}

The aim for this special issue was to target research on translation, with an emphasis on behavior change processes - as opposed to compiling evidence of a specific behavioral effect or outcome. As a result, the response to the special issue call resulted in a broad range of studies conducted across diverse populations engaging in a variety of physical activity behaviors. Within this issue, you will find scoping reviews, qualitative investigations, largescale epidemiological correlational studies, intervention development descriptions, and pilot trial efficacy findings. The evidence originates from high, middle, and low-income countries and spans clinical and non-clinical adult populations including people with depression, people with severe mental illness, women in early alcohol recovery programs, and people in prisons. The studies investigated demographic correlates, psychosocial determinants, and retrospective insight into people's experiences with physical activity. Additionally, this issue considers a breadth of physical activity behaviors, defined as any physical movement, including specific types of activity like exercise and sport (Caspersen, Powell, \& Christenson, 1985). Within the issue, physical activity includes structured, supervised exercise programmers, and unstructured physical activity obtained through occupational or domestic activities, leisure physical activity, active commuting, and sport participation.

Although the origins, populations, and study designs within this special issue are richly diverse, the findings lend themselves to an elegant overarching message - people with 
mental health issues have unique facilitators and barriers to engaging in physical activity. The articles within this issue exemplify some unique moderators of behavior change processes within people with mental health issues. This compilation of evidence speaks toward the need to make meaningful changes at individual and system levels to increase physical activity in people with mental health issues worldwide.

In their qualitative exploration, Hargreaves, Lucock, and Rodriguez (2017 - this issue) highlights the individuality of physical activity behavior change processes in people with severe mental illness and the dynamic nature of the motivational processes at play while people undergo recovery. People in the study reported that professional support and accessibility was important for the initiation of physical activity, but that having individually meaningful physical activity experiences were important for maintenance. These study findings suggest that one-size-fits-all interventions may not be suitable for people with mental health issues. To be effective, interventions may need to be adaptable to individual differences and the dynamic nature of mental illness symptoms, responses to medication, physical activity motivation, and ongoing mental health recovery processes.

However, Vancampfort, De Hert and colleagues' (2017 - this issue) investigation of physical activity motives of Ugandan outpatients with psychosis suggests that some motives for physical activity behavior change may not necessarily be dependent on variations in behavior change processes. Improvements in fitness and interest/enjoyment of physical activity were found to be the primary motives for both men and women, irrespective of symptom severity. Notably, the findings suggest that motives were not different between those who had recently begun being active (i.e., in the action stage of change) and those who had been active for at least six months (i.e., in the maintenance stage of change). It may be that physical activity motives do not change across the physical activity behavior change processes of initiation and maintenance. However, these conclusions have to be tempered 
until these processes can be tracked longitudinally across these processes of change. This study adds to previous evidence suggesting that the stage categorization of behavior change processes put forth by the Transtheoretical Model (Prochaska \& DiClemente, 1982) may not effectively map onto physical activity behavior change processes (Bridle et al., 2005; Povey, Conner, Sparks, James, \& Shepherd, 1999; van Sluijs, van Poppel, \& van Mechelen, 2004; West, 2005). It is likely that physical activity behavior change processes evolve not as abrupt stages but rather as gradual shifts (West, 2005).

In addition to considering if motivation may evolve over the physical activity behavior change process, evidence from this issue suggests that physical activity interventions for people with mental health issues need to be multi-faceted. Chen and colleagues (2017 - this issue) conducted a qualitative study of community-dwelling people with severe mental illness in Taiwan. The facilitators and barriers faced by this population were multi-dimensional and included many of the personal, social, and environmental factors also found to be important amongst the general population (e.g., social support, self-efficacy, accessibility to facilities). However, it was also revealed that this group faces a barrier to physical activity unique from the general population - a fear of stigma associated with mental illness. Negative anticipation about physical activity experiences can be detrimental to motivation for future physical activity (Williams \& Evans, 2014), and the evidence being exposed through this issue suggests that this barrier may be particularly influential amongst people with mental health issues.

Adding further credence to this argument is Glowacki, Duncan, Gainforth, and Faulkner's (2017 - this issue) article in this issue involving a scoping review of the barriers and facilitators of physical activity among people with depression. These researchers structured their findings within the theoretical domains framework (Cane, O'Connor, \& Michie, 2012), showing that most barriers and facilitators were similar to those of general 
populations, but that people with depression seemed to be particularly reliant on behavior change processes within the Emotion domain (e.g., negative emotions, outcome expectations, deprecating self-assessment). The authors rightly point out that most traditional behavior change theories provide little guidance for the impact of emotion on behavior change processes or how it may be effectively managed or targeted in interventions. The insights gained from this review imply that traditional behavior change theories are likely not sufficient for promoting physical activity amongst people with mental health issues.

One strategy for overcoming emotion-related barriers to physical activity behavior change is behavioral activation. Behavioral activation is a treatment for depression in which people are aided in overcoming symptoms of avoidance and social withdrawal through scheduling positive daily life activities (Jacobson, Martell, \& Dimidjian, 2001), rather than focusing on influencing changes in cognitions. Lambert et al. (2017 - this issue) describes their application of behavioral activation in their web-based intervention, eMotion, to promote physical activity in people with depression. The development and theoretical basis of their self-delivered intervention is systematically described in detail based on the Centre for eHealth Research and Disease Management roadmap (van Gemert-Pijnen et al., 2011). Intervention mapping accounts such as these provide accountability and transparency into the intervention design process, which the authors note is generally lacking across psychological interventions. This trial speaks to the potential for utilizing mental illness therapy strategies to promote physical activity.

Evidence from a study within this issue also suggests that physical activity promotion may aid in enhancing non-activity therapy strategies. In a pilot study, Blevins et al. (2017 this issue) tracked motivation and beliefs about exercise among women in early recovery from alcohol across a lifestyle physical activity intervention. They showed that autonomous motivation for physical activity changed across the intervention, but beliefs about exercise 
did not. Across the intervention, women who increased their physical activity as a means to cope with negative affect and cravings more often utilized adaptive coping strategies other than physical activity as well, including seeking emotional support from others. The link between physical activity and alcohol consumption is complex (Conroy, Pincus, Ram, \& al'Absi, 2017), but these findings imply that there is potential for physical activity programs to be a means for delivering psychosocial treatment strategies for alcohol recovery, such as social support.

Additional articles within this issue provide insight into how interventions may be structured to optimize effectiveness amongst people with mental health issues. Chang and colleagues (2017 - this issue) present pilot data comparing one-way and two-way text messaging behavior change strategies across a 12-week intervention promoting physical activity among people with severe mental illness. Whereas the one-way messages were simple notifications, the two-way messages required participants to respond, thereby initiating attention and interaction with the intervention content. Although underpowered for testing between group differences, the trends suggest that two-way messaging may be more effective amongst this population. In general populations, encouraging participants to interact and engage with intervention content can aid in behavior change (Short, Rebar, Plotnikoff, \& Vandelanotte, 2015); it seems that intervening with physical activity amongst people with mental health issues is no exception to this need to attend to and monitor engagement.

This issue also provides evidence that physical activity promotion may enhance mental health in specific populations at risk for mental health issues. In their review of the impact of sport-based interventions on psychological wellbeing of people in prison, Woods, Breslin, and Hassan (2017 - this issue) found that there is potential for physical activity interventions to enhance mental health in prison populations, but highlighted that the use of health behavior change theories was notably absent amongst the study descriptions. 
Individuals within prison settings have unique constraints on their choices and behaviors that may alter behavior change processes (e.g., autonomy, perceived behavioral control). Based on those insights, the authors of the review note how important it is for research to investigate the psychological processes at play in physical activity promotion amongst people in this unique setting. This review also brought to light the difficulties of conducting rigorous trials within controlled settings such as prisons. Conducting rigorous controlled trials in contexts such as prisons or residential psychiatric care settings is undoubtedly challenging (Firth et al., 2017), but these barriers cannot excuse researchers from conducting the rigorous, theorydriven science that these at-risk populations deserve.

Other evidence from studies housed within this issue highlights the importance that societal, cultural, and national contexts can have on behavior change processes and how system-level changes may be required to make regular physical activity engagement more achievable amongst particular populations of people with mental health issues. Vancampfort, Stubbs, Hallgren, and Koyanagi (2017 - this issue) compared demographic profiles of people with hazardous alcohol use across 46 low- and middle-income countries, finding that people were more likely to be inactive if they were older, had higher incomes, were more educated, unemployed, lived in urban settings, and had mobility difficulties. The authors suggest that system-level regulations to increase the safety and accessibility of physical activity opportunities in urban settings amongst these countries may be needed to enhance physical activity amongst these populations.

Rezaie, Shafaroodi, \& Philips (2017 - this issue) - a qualitative study of the barriers to physical activity amongst Iranian women psychiatric ward patients - found that, alongside the barriers of mental illness such as anticipated stigma and medication side effects, these women perceived cultural barriers such as anticipated negative attitudes toward women exercising and negative views of group-based exercise. Physical activity interventions for 
people with mental health issues will need to be considerate of the contextual barriers that may impede physical activity behavior change processes, some of which may be exacerbated by poor mental health.

\section{Conclusions \& Reflections}

This special issue demonstrates that people with mental health issues have unique facilitators and barriers to physical activity, which change over time, and are dependent on contextual and person factors. These articles serve to complement the existing strong evidence for the effectiveness of physical activity for benefiting mental health clinical (e.g., Bailey et al., 2017; Rebar, Stanton, et al., 2015; Rosenbaum et al., 2014), in that they highlight factors that may impact efficacy. We encourage researchers to focus more on translation and the factors that may influence it. From the findings within this issue, it seems clear that a generic prescription for physical activity is not sufficient to elicit mental health benefits, but rather should be tailored to maximize people's opportunities, willingness, and commitment to engage in physical activity. Interventions that worked for some people once may not work for other people or even the same people at a different time.

Informed by the evidence within this issue, Figure 1 illustrates how prescribing physical activity does not directly lead to mental health and wellbeing benefits. Rather, the efficacy of these efforts are entirely reliant on a person's current states, past, and context. Mental health issues reverberate across these different layers of impact on behavior change. For example, this issue demonstrated that physical activity of people with, or at high risk for mental health issues, is influenced by contextual factors such as cultural expectations (Rezaie et al., 2017 - this issue) and accessibility to physical activity (Hargreaves et al., 2017 - this issue; Woods et al., 2017 - this issue), demographic factors such as income and education (Vancampfort, Stubbs, et al., 2017 - this issue), past experiences with physical activity (Vancampfort, De Hert, et al., 2017 - this issue), previous mental health treatment (Chen et 
al., 2017 - this issue), and current states including anticipation about the behavior (Glowacki et al., 2017 - this issue), emotions and mood (Glowacki et al., 2017 - this issue), concurrent physical and mental health treatment (Blevins et al., 2017 - this issue), and motivational states (Blevins et al., 2017 - this issue; Chen et al., 2017 - this issue; Vancampfort, De Hert, et al., 2017 - this issue).

What we are proposing is not new; indeed the call for behavior change intervention translation to address system and individual factors has a long history (e.g., Biddle \& Mutrie, 2007; Engel, 1980; Sniehotta et al., 2017). Rather, this issue should serve as a reminder that the science of mental health and physical activity is not unidirectional, and should not only be studied as an isolated cause of behavior on a mental health outcome, but as a reciprocal process that changes over time and differs between people and contexts.

Much of the existing evidence of physical activity and mental health is based on the prescription of supervised, easily accessible exercise programs performed by a convenient and willing/compliant sample for a certain duration. Indeed, the United Kingdom's National Institute of Health and Care Excellence (NICE, 2009), drawing on available evidence, simply recommend people with mild or moderate depression should receive group-based exercise, with support from a competent practitioner, across three sessions per week of moderate duration (45 min - 1 hour) over 10-14 weeks. Guideline-based recommendations like these are clearly based on an assumption that fitness translates into reduced depression symptoms, neglecting the acute affective and motivational benefits of physical activity (e.g., Bartholomew, Morrison, \& Ciccolo, 2005; Bodin \& Martinsen, 2004; Ekkekakis, 2003; Rebar, Faulkner, \& Stanton, 2015; Reed \& Ones, 2006; Williams, 2008).

One risk of generic prescriptions like these is that we will undermine people's motivation to be physically active in the future. Some mental health issues can elicit heightened self-focused attention (Ingram, 1990), negative self-beliefs (Taylor \& Brown, 
1988), and negative reactions to failure (Johnstone, Reekum, Urry, Kalin, \& Davidson, 2007). Under these circumstances, if physical activity prescriptions seem impossible to achieve or result in people experiencing failure, it can undermine competence and future willingness to try physical activity again (Wrosch, Scheier, Miller, Schulz, \& Carver, 2003). Additionally, exerting external control over individuals' physical activity decisions with specific exercise prescriptions may also undermine their sense of control and autonomy, thereby reducing their intrinsic motivation for physical activity and, with it, the chance that they will engage in

physical activity for more than a few months (Deci \& Ryan, 2002; Teixeira, Carraça, Markland, Silva, \& Ryan, 2012). We need to extend our line of inquiry into the efficacy and consequences of generic physical activity prescriptions, such as those recommended by national guidelines.

\section{Structured Call for Future Research}

When it comes to practically implementing specific prescriptions for people with mental health issues, questions raise about patient acceptability (what do we do if the patients are not willing?), maintenance (what happens after those 14 weeks?), scalability (how can we reach everyone in need?) and generalizability (will this work outside of the controlled settings?). These practical implementation issues must drive our future research.

Acceptability. People with mental health issues may be at a particular risk for dropping out in physical activity programs (e.g., Cooney et al., 2013; Tobi, Kemp, \& Schmidt, 2017). This issue must be addressed within the field. Future research should investigate what predicts acceptability of physical activity programs in people with mental health issues and how to effectively engage these populations in physical activity interventions. As an example, the BAcPAc trial (Farrand et al., 2014; Pentecost et al., 2015) aimed to maximize acceptability by shifting the focus away from explicitly promoting exercise to a more subtle 
approach to encouraging behaviors that involved physical activity that may have been lost as a result of depression.

Maintenance. Alongside the issue of acceptability is the problem of poor behavioral change maintenance. Most physical activity interventions targeted toward people with mental health issues have a distinct endpoint, but mental health benefits of physical activity will be maximized when activity is maintained long-term. Further investigation is needed into what happens to people's physical activity following prescribed activity programs and how to aide people in maintaining regular activity outside of prescribed programs. Given that many mental health issues are associated with deregulation of the brain systems associated with reinforcement learning (e.g., Byrne, Patrick, \& Worthy, 2016; Chen, Takahashi, Nakagawa, Inoue, \& Kusumi, 2015; Slifstein et al., 2015), more is needed to understand how physical activity habit formation may be influenced by mental health issues and whether specific habit formation behavior change strategies are more effective in enhancing physical activity amidst this population.

Scalability. The evidence within this special issue illustrates the important point that implementing interventions and treatment in real-world settings is multi-faceted and requires change at an individual, organizational and/or community levels (Michie, Atkins, \& West, 2014). The planning and implementation of physical activity interventions for people with mental health issues need to be considerate of how implementation can be scaled beyond single sites or controlled environments. The paper by Lambert et al (2017 - this issue) highlights one such intervention delivered online as a way to broaden community reach.

Generalizability. Many of the physical activity interventions for people with or atrisk for mental health issues are not generalizable simply because they are not described in a way that is easily replicable. Contemporary research involving complex interventions (Medical Research Council, 2006) requires much greater detail on the components included 
in the intervention mapped against theoretical domains and behavior change processes, following the TiDIER guidelines (http://www.equator-network.org/reportingguidelines/tidier/). If the science of understanding how to support those with mental health issues is to advance, there must be more transparency in our study reporting to enable duplication, and advance our understanding about which intervention aspects are sufficient and necessary for effectiveness and efficacy. Mental health and physical activity researchers conducting intervention trials should build in appropriate mixed methods process evaluation (Moore et al., 2015) so that the trial outcomes add knowledge about how participants experience interventions, the level of intervention fidelity (Lambert et al., 2017), and what techniques are most effective in overcoming some of the barriers and facilitators identified in the papers in this issue.

\section{Important Note about Sedentary Behavior}

In contrast to physical activity, sedentary behavior is any waking behavior characterized by an energy expenditure $\leq 1.5$ metabolic equivalents (METs), while in a sitting, reclining or lying posture, including sitting, and lying down (Barnes et al., 2012; Tremblay et al., 2017). Given the growing investigations into the link between sedentary behavior and mental health (Faulkner \& Biddle, 2013), the aim of this issue was also to highlight investigations into sedentary behavior in people with mental health issues; however none of the articles specifically focused on sedentary behavior. Alongside research into the interplay between physical activity and sedentary behavior and their impacts on mental health, it will be important for future research to consider how mental health issues may interfere with sedentary behavior change processes.

\section{Conclusions}

In summary, this special issue illustrates the importance for mental health and physical activity research to reach beyond investigations of direct associations between 
physical activity behavior and mental health outcomes. People with mental health issues have unique facilitators and barriers to physical activity that may impact the circumstances under which interventions will or will not be effective. We need scientific advancements on translation of physical activity trials for people with mental health issues to address existing gaps in knowledge regarding acceptability, maintenance, scalability and generalizability. As part of this process, it is important that the field further investigate the impact of mental health issues on behavior change processes. We are calling for researchers to provide transparent descriptions of efficacy trials which are: 1 - adaptable across the unique contextual situations of individuals and the dynamic nature of behavior change processes, 2 theory-driven, thereby targeting behavior change processes as opposed to a series of isolate constructs (Michie \& Abraham, 2004), and 3 - multi-faceted, ideally through a multidisciplinary approach to behavior change (Vancampfort \& Faulkner, 2014). 


\section{References}

Bailey, A. P., Hetrick, S. E., Rosenbaum, S., Purcell, R., \& Parker, A. G. (2017). Treating depression with physical activity in adolescents and young adults: A systematic review and meta-analysis of randomised controlled trials. Psychological Medicine, 120.

Bardes, C. L. (2012). Defining “patient-centered medicine.” New England Journal of Medicine, 366(9), 782-783.

Barnes, J., Behrens, T. K., Benden, M. E., Biddle, S., Bond, D., Brassard, P., ... Network, S. B. R. (2012). Letter to the Editor: Standardized use of the terms "sedentary" and “sedentary behaviours." Applied Physiology Nutrition and Metabolism-Physiologie Appliquee Nutrition Et Metabolisme, 37(3), 540-542. https://doi.org/10.1139/H2012024

Bartholomew, J. B., Morrison, D., \& Ciccolo, J. T. (2005). Effects of acute exercise on mood and well-being in patients with major depressive disorder. Medicine \& Science in Sports \& Exercise, 37(12), 2032-2037.

Biddle, S. J., \& Mutrie, N. (2007). Psychology of physical activity: Determinants, well-being and interventions. London and New York: Routledge.

Blevins, C., Rapoport, M., Battle, C., Stein, M., Abrantes, A., Wilson, P., \& Fortier, M. (2017 - this issue). Changes in coping, autonomous motivation, and beliefs about exercise among women in early recovery from alcohol participating in a lifestyle physical activity intervention. Mental Health and Physical Activity.

Bodin, T., \& Martinsen, E. W. (2004). Mood and self-efficacy during acute exercise in clinical depression. A randomized, controlled Study. Journal of Sport and Exercise Psychology, 26(4), 623-633. https://doi.org/10.1123/jsep.26.4.623 
Bridle, C., Riemsma, R. P., Pattenden, J., Sowden, A. J., Mather, L., Watt, I. S., \& Walker, A. (2005). Systematic review of the effectiveness of health behavior interventions based on the transtheoretical model. Psychology \& Health, 20(3), 283-301. https://doi.org/10.1080/08870440512331333997

Byrne, K. A., Patrick, C. J., \& Worthy, D. A. (2016). Striatal dopamine, externalizing proneness, and substance abuse: Effects on wanting and learning during reward-based decision making. Clinical Psychological Science, 4(5), 760-774.

Cane, J., O’Connor, D., \& Michie, S. (2012). Validation of the theoretical domains framework for use in behaviour change and implementation research. Implementation Science, 7, 37. https://doi.org/10.1186/1748-5908-7-37

Caspersen, C. J., Powell, K. E., \& Christenson, G. M. (1985). Physical activity, exercise, and physical fitness: Definitions and distinctions for health-related research. Public Health Reports, 100(2), 126-131.

Chen, M.-D., Chang, J.-J., Kuo, C.-C., Yu, J.-W., Huang, M.-F., Marks, B., \& Chang, Y.-C. (2017 - this issue). A pilot comparative study of one-way versus two-way text message program to promote physical activity among people with severe mental illness. Mental Health and Physical Activity.

Chen, C., Takahashi, T., Nakagawa, S., Inoue, T., \& Kusumi, I. (2015). Reinforcement learning in depression: A review of computational research. Neuroscience \& Biobehavioral Reviews, 55(Supplement C), 247-267. https://doi.org/10.1016/j.neubiorev.2015.05.005

Cooney, G. M., Dwan, K., Greig, C. A., Lawlor, D. A., Rimer, J., Waugh, F. R., ... Mead, G. E. (2013). Exercise for depression. Cochrane Database Syst Rev, 12(9).

Deci, E. L., \& Ryan, R. M. (2002). Handbook of self-determination research. New York: University Rochester Press. 
Ekkekakis, P. (2003). Pleasure and displeasure from the body: Perspectives from exercise. Cognition \& Emotion, 17(2), 213-239.

Engel, G. L. (1980). The clinical application of the biopsychosocial model. American Journal of Psychiatry, 137(5), 535-544.

Farholm, A., \& Sørensen, M. (2016). Motivation for physical activity and exercise in severe mental illness: A systematic review of intervention studies. International Journal of Mental Health Nursing, 25(3), 194-205.

Farrand, P., Pentecost, C., Greaves, C., Taylor, R. S., Warren, F., Green, C., ... Taylor, A. H. (2014). A written self-help intervention for depressed adults comparing behavioural activation combined with physical activity promotion with a self-help intervention based upon behavioural activation alone: study protocol for a parallel group pilot randomised controlled trial (BAcPAc). Trials, 15(1), 196-207. https://doi.org/10.1186/1745-6215-15-196

Faulkner, G., \& Biddle, S. (2013). Standing on top of the world: Is sedentary behaviour associated with mental health? Mental Health and Physical Activity, 6(1), 1-2.

Firth, J., Carney, R., Pownall, M., French, P., Elliott, R., Cotter, J., \& Yung, A. R. (2017). Challenges in implementing an exercise intervention within residential psychiatric care: A mixed methods study. Mental Health and Physical Activity, 12, 141-146.

Glowacki, K., Duncan, M., Gainforth, H., Faulkner, G. (2017 - this issue). Barriers and facilitators to physical activity and exercise among adults with depression: A scoping review. Mental Health and Physical Activity.

Hargreaves, J., Lucock, M., \& Rodriguez, A. (2017 - this issue). From inactivity to becoming physically active: The experiences of behaviour change in people with serious mental illness. Mental Health and Physical Activity. 
Ingram, R. E. (1990). Self-focused attention in clinical disorders: Review and a conceptual model. Psychological Bulletin, 107(2), 156-176. http://dx.doi.org/10.1037/00332909.107.2.156

Jacobson, N. S., Martell, C. R., \& Dimidjian, S. (2001). Behavioral activation treatment for depression: Returning to contextual roots. Clinical Psychology: Science and Practice, 8(3), 255-270. https://doi.org/10.1093/clipsy.8.3.255

Johnstone, T., Reekum, C. M. van, Urry, H. L., Kalin, N. H., \& Davidson, R. J. (2007). Failure to regulate: Counterproductive recruitment of top-down prefrontal-subcortical circuitry in Major Depression. Journal of Neuroscience, 27(33), 8877-8884. https://doi.org/10.1523/JNEUROSCI.2063-07.2007

Chen, M.-D., I, J.-H., Pellegrini, C., Tang, T.-C., \& Kuo, C.-C. (2017 - this issue). A qualitative exploration of facilitators and barriers to physical activity participation in people with severe mental illness in Taiwan. Mental Health and Physical Activity.

Lambert, J. D., Greaves, C. J., Farrand, P., Cross, R., Haase, A. M., \& Taylor, A. H. (2017). Assessment of fidelity in individual level behaviour change interventions promoting physical activity among adults: a systematic review. BMC Public Health, 17(1), 765777. https://doi.org/10.1186/s12889-017-4778-6

Medical Research Council. (2006). Developing and evaluating complex interventions: New guidance. London: Medical Research Council.

Michie, S., \& Abraham, C. (2004). Interventions to change health behaviours: Evidencebased or evidence-inspired? Psychology \& Health, 19(1), 29-49. https://doi.org/10.1080/0887044031000141199

Moore, G. F., Audrey, S., Barker, M., Bond, L., Bonell, C., Hardeman, W., ... \& Baird, J. (2015). Process evaluation of complex interventions: Medical Research Council guidance. $B M J, 350$, h1258. https://doi.org/10.1136/bmj.h1258 
National Institute for Health and Clinical Excellence. (2009). Depression: Treatment and management of depression in adults: Clinical guideline 90. London: NICE.

Pentecost, C., Farrand, P., Greaves, C. J., Taylor, R. S., Warren, F. C., Hillsdon, M., ... Evans, P. H. (2015). Combining behavioural activation with physical activity promotion for adults with depression: findings of a parallel-group pilot randomised controlled trial (BAcPAc). Trials, 16(1), 367-382. https://doi.org/10.1186/s13063015-0881-0

Povey, R., Conner, M., Sparks, P., James, R., \& Shepherd, R. (1999). A critical examination of the application of the Transtheoretical Model's stages of change to dietary behaviours. Health Education Research, 14(5), 641-651. https://doi.org/10.1093/her/14.5.641

Prochaska, J. O., \& DiClemente, C. C. (1982). Transtheoretical therapy: Toward a more integrative model of change. Psychotherapy: Theory, Research \& Practice, 19(3), $276-288$.

Rebar, A. L., Faulkner, G., \& Stanton, R. (2015). An exploratory study examining the core affect hypothesis of the anti-depressive and anxiolytic effects of physical activity. Mental Health and Physical Activity, 9, 55-58. https://doi.org/10.1016/j.mhpa.2015.10.001

Rebar, A. L., Stanton, R., Geard, D., Short, C., Duncan, M. J., \& Vandelanotte, C. (2015). A meta-meta-analysis of the effect of physical activity on depression and anxiety in nonclinical adult populations. Health Psychology Review, 9(3), 366-378. https://doi.org/10.1080/17437199.2015.1022901

Reed, J., \& Ones, D. S. (2006). The effect of acute aerobic exercise on positive activated affect: A meta-analysis. Psychology of Sport and Exercise, 7(5), 477-514. https://doi.org/10.1016/j.psychsport.2005.11.003 
Rezaie, L., Shafaroodi, N., \& Philips, D. (2017 - this issue). The barriers to participation in leisure time physical activities among women with severe mental illness: A qualitative study. Mental Health and Physical Activity.

Roessler, K. K., Bramsen, R. H., Dervisevic, A., \& Bilberg, R. (2017). Exercise based interventions for alcohol use disorder: A comment on motivational aspects of participation. Scandinavian Journal of Psychology, 58(1), 23-28.

Rosenbaum, S., Tiedemann, A., Sherrington, C., Curtis, J., \& Ward, P. B. (2014). Physical activity interventions for people with mental illness: A systematic review and metaanalysis. The Journal of Clinical Psychiatry, 75(9), 964-974.

Schuch, F., Vancampfort, D., Firth, J., Rosenbaum, S., Ward, P., Reichert, T., ... Stubbs, B. (2017). Physical activity and sedentary behavior in people with major depressive disorder: a systematic review and meta-analysis. Journal of Affective Disorders, 210, $139-150$.

Short, C. E., Rebar, A. L., Plotnikoff, R. C., \& Vandelanotte, C. (2015). Designing engaging online behaviour change interventions: A proposed model of user engagement. European Health Psychologist, 17(1), 32-38.

Slifstein, M., van de Giessen, E., Van Snellenberg, J., Thompson, J. L., Narendran, R., Gil, R., ... Moore, H. (2015). Deficits in prefrontal cortical and extrastriatal dopamine release in schizophrenia: a positron emission tomographic functional magnetic resonance imaging study. JAMA Psychiatry, 72(4), 316-324.

Sniehotta, F. F., Araújo-Soares, V., Brown, J., Kelly, M. P., Michie, S., \& West, R. (2017). Complex systems and individual-level approaches to population health: a false dichotomy? The Lancet Public Health, 2(9), e396-e397. https://doi.org/10.1016/S2468-2667(17)30167-6 
Taylor, S. E., \& Brown, J. D. (1988). Illusion and well-being: A social psychological perspective on mental health. Psychological Bulletin, 103(2), 193-210. http://dx.doi.org/10.1037/0033-2909.103.2.193

Teixeira, P. J., Carraça, E. V., Markland, D., Silva, M. N., \& Ryan, R. M. (2012). Exercise, physical activity, and self-determination theory: A systematic review. International Journal of Behavioral Nutrition and Physical Activity, 9(1), 78-107. https://doi.org/10.1186/1479-5868-9-78

Tobi, P., Kemp, P., \& Schmidt, E. (2017). Cohort differences in exercise adherence among primary care patients referred for mental health versus physical health conditions. Primary Health Care Research \& Development, 18(5), 463-471.

Tremblay, M. S., Aubert, S., Barnes, J. D., Saunders, T. J., Carson, V., Latimer-Cheung, A. E., ... Chinapaw, M. J. M. (2017). Sedentary Behavior Research Network (SBRN) Terminology Consensus Project process and outcome. International Journal of Behavioral Nutrition and Physical Activity, 14, 75-91. https://doi.org/10.1186/s12966-017-0525-8

van Gemert-Pijnen, J. E., Nijland, N., van Limburg, M., Ossebaard, H. C., Kelders, S. M., Eysenbach, G., \& Seydel, E. R. (2011). A holistic framework to improve the uptake and impact of eHealth technologies. Journal of Medical Internet Research, 13(4). e111. https://doi.org/10.2196/jmir.1672

van Sluijs, E. M. F., van Poppel, M. N. M., \& van Mechelen, W. (2004). Stage-based lifestyle interventions in primary care: Are they effective? American Journal of Preventive Medicine, 26(4), 330-343. https://doi.org/10.1016/j.amepre.2003.12.010

Vancampfort, D., De Hert, M., Probst, M., Firth, J., Myin-Germeys, van Winkel...Mugisha, J. (2017 - this issue). Interest, competence, appearance, fitness and social relatedness as 
motives for physical activity in Ugandan outpatients with psychosis. Mental Health and Physical Activity.

Vancampfort, D., \& Faulkner, G. (2014). Physical activity and serious mental illness: A multidisciplinary call to action. Mental Health and Physical Activity, 7(3), 153-154. https://doi.org/10.1016/j.mhpa.2014.11.001

Vamcampfort, D., Stubbs, B., Hallgren, M., \& Koyanagi, A. (2017 - this issue). Physical activity correlates in people with hazardous alcohol use: Data from 46 low- and middle-income countries. Mental Health and Physical Activity.

West, R. (2005). Time for a change: Putting the Transtheoretical (Stages of Change) Model to rest. Addiction, 100(8), 1036-1039. https://doi.org/10.1111/j.13600443.2005.01139.x

Williams, D. M. (2008). Exercise, affect, and adherence: An integrated model and a case for self-paced exercise. Journal of Sport and Exercise Psychology, 30(5), 471-496. https://doi.org/10.1123/jsep.30.5.471

Williams, D. M., \& Evans, D. R. (2014). Current emotion research in health behavior science. Emotion Review, 6(3), 277-287. https://doi.org/10.1177/1754073914523052

Woods, D., Breslin, G., \& Hassan, D. (2017 - this issue). A systematic review of the impact of sport-based interventions on the psychological well-being of people in prison. Mental Health and Physical Activity.

World Health Organization. (2017). Mental health included in the UN Sustainable Development Goals. Geneva, Switzerland.

Wrosch, C., Scheier, M. F., Miller, G. E., Schulz, R., \& Carver, C. S. (2003). Adaptive selfregulation of unattainable goals: Goal disengagement, goal reengagement, and subjective well-being. Personality and Social Psychology Bulletin, 29(12), 14941508. https://doi.org/10.1177/0146167203256921 


\section{Figure Captions}

Figure 1. An illustration of how prescriptions for physical activity do not directly lead to mental health and wellbeing benefits, but rather are entirely dependent on a person's uptake of the behaviour, which is dependent on their current state, past and context.

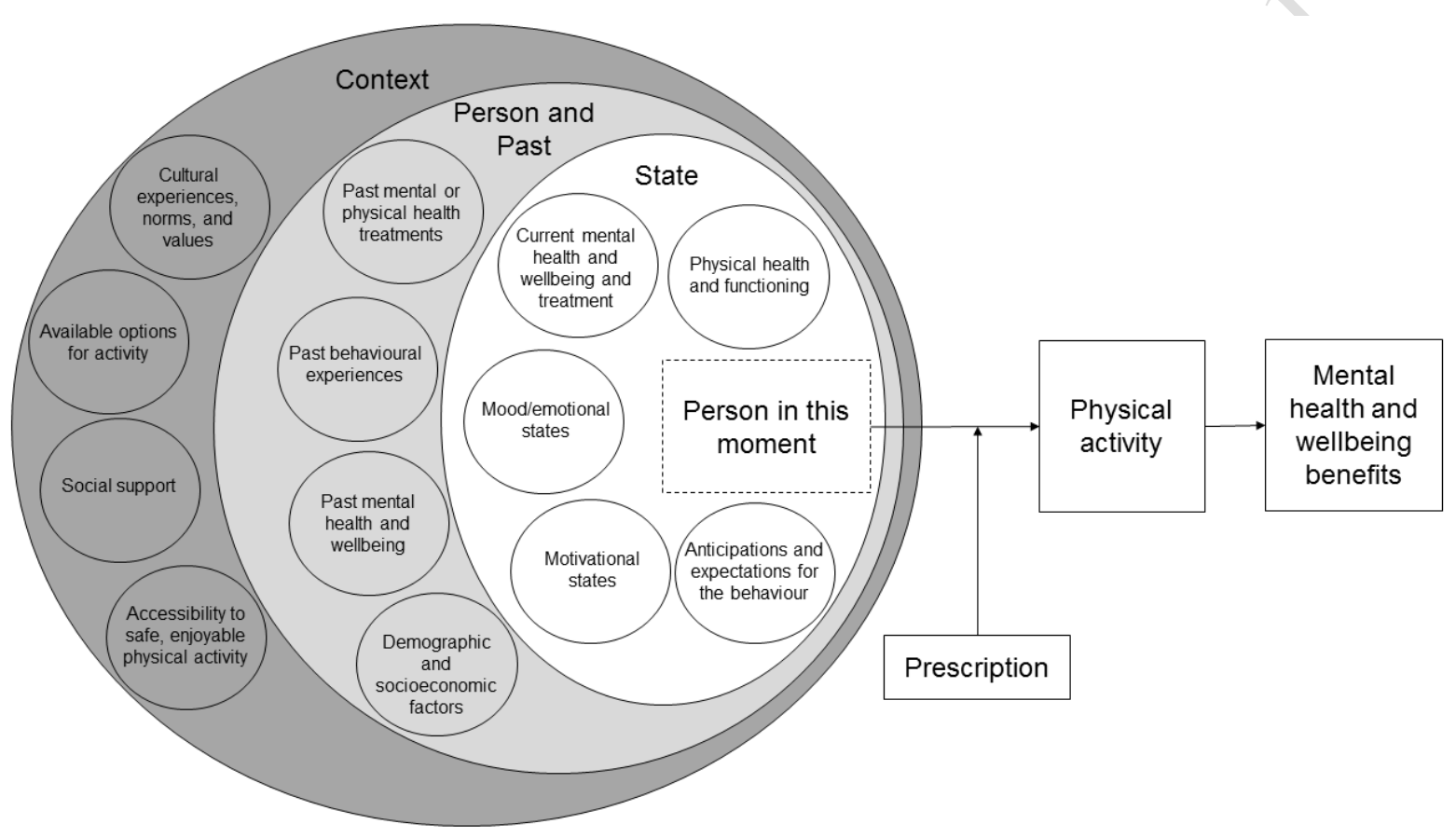

